\title{
Acute consumption of a caffeinated energy drink enhances aspects of performance in sprint swimmers
}

\author{
Beatriz Lara, Diana Ruiz-Vicente, Francisco Areces, Javier Abián-Vicén, Juan José Salinero, \\ Cristina Gonzalez-Millán, César Gallo-Salazar and Juan Del Coso* \\ Exercise Physiology Laboratory, Camilo José Cela University, Madrid 28692, Spain
}

(Submitted 9 July 2014 - Final revision received 12 January 2015 - Accepted 5 June 2015 - First published online 17 August 2015)

\begin{abstract}
This study investigated the effect of a caffeinated energy drink on various aspects of performance in sprint swimmers. In a randomised and counterbalanced order, fourteen male sprint swimmers performed two acute experimental trials after the ingestion of a caffeinated energy drink $(3 \mathrm{mg} / \mathrm{kg})$ or after the ingestion of the same energy drink without caffeine $(0 \mathrm{mg} / \mathrm{kg}$; placebo). After 60 min of ingestion of the beverages, the swimmers performed a countermovement jump, a maximal handgrip test, a $50 \mathrm{~m}$ simulated competition and a $45 \mathrm{~s}$ swim at maximal intensity in a swim ergometer. A blood sample was withdrawn $1 \mathrm{~min}$ after the completion of the ergometer test. In comparison with the placebo drink, the intake of the caffeinated energy drink increased the height in the countermovement jump (49.4 (SD 5.3) $v$. 50.9 (SD 5.2) cm, respectively; $P<0.05$ ) and maximal force during the handgrip test with the right hand (481 (SD 49) $v .498$ (SD 43) N; $P<0.05$ ). Furthermore, the caffeinated energy drink reduced the time needed to complete the $50 \mathrm{~m}$ simulated swimming competition (27.8 (SD 3.4) $v$. 27.5 (sD 3.2) s; $P<0.05$ ), and it increased peak power (273 (SD 55) $v .303$ (SD 49) W; $P<0.05$ ) and blood lactate concentration (11.0 (SD 2.0$) v .11 .7$ (SD 2.1$) \mathrm{mm}$; $P<0.05)$ during the ergometer test. The caffeinated energy drink did not modify the prevalence of insomnia ( $7 v .7 \%)$, muscle pain $(36 v$. $36 \%)$ or headache $(0 v .7 \%)$ during the hours following its ingestion $(P>0.05)$. A caffeinated energy drink increased some aspects of swimming performance in competitive sprinters, whereas the side effects derived from the intake of this beverage were marginal at this dosage.
\end{abstract}

Key words: Sprint: Swimming: Nutrition: Exercise: Dietary supplements: Ergogenic aid

Caffeine-containing energy drinks are relatively new commercially available beverages intended to increase activeness, vigour and overall performance in situations of physical and mental stress. These drinks contain different amounts of carbohydrates, taurine and group B vitamins, although the ingredient of current interest is caffeine $^{(1)}$. In the sport setting, energy drinks have become one of the most used supplements among young and elite athletes ${ }^{(2)}$, probably because of the well-recognised ergogenicity of caffeine. The popularity of caffeine and caffeine-containing products has been also increased because this substance is no longer considered a doping substance ${ }^{(3)}$. Although the use of caffeine in sports was considered doping from 1984 to 2004 (when athletes' urinary caffeine concentration exceeded $12 \mu \mathrm{g} / \mathrm{ml}$ ), caffeine was placed on the World Antidoping Monitoring Program in 2004, to track the trends of its use and to assess its future re-inclusion in the banned list ${ }^{(4)}$.

Several investigations have determined the ergogenicity of energy drinks during soccer ${ }^{(1,5)}$, basketball $^{(6)}$, volleyball ${ }^{(7)}$ and rugby competitions ${ }^{(8,9)}$ when ingested in an amount equivalent to providing $3 \mathrm{mg}$ of caffeine/ $\mathrm{kg}$ of body mass. Interestingly, the ergogenicity of caffeinated energy drinks is not present when the amount of energy drink provides only $1 \mathrm{mg} / \mathrm{kg}$ caffeine $^{(10,11)}$. All these investigations have been carried out during team sports matches that combine bouts of high-intensity exercise with periods of lower intensity or recovery during a relatively long period of time. Nevertheless, the results obtained with these team-sports players can hardly be used to infer the effect of caffeine in other continuous and power-based sports such as swimming. Moreover, the scientific information about the ergogenicity of caffeine in swimming is very limited and contradictory.

Collomp et al. ${ }^{(12)}$ provided $250 \mathrm{mg}$ of caffeine or a placebo substance to trained and untrained swimmers before a $2 \times 100 \mathrm{~m}$ freestyle time trial. After the ingestion of the caffeine, trained swimmers reduced the time needed to complete the trial by approximately $2 \%$, but this performance benefit was not evident in the untrained swimmers. However, both groups of swimmers presented higher post-exercise blood lactate concentrations with the ingestion of caffeine when compared with the placebo. MacIntosh \& Wright ${ }^{(13)}$ evaluated the benefits obtained after the ingestion of $6 \mathrm{mg} / \mathrm{kg}$ of caffeine during a $1500 \mathrm{~m}$ swim-time trial. Swimmers reduced their test time by $1.8 \%$ with the ingestion of caffeine, and this performance benefit was accompanied by higher levels of blood glucose

*Corresponding author: J. Del Coso, fax +34 918153 131, email jdelcoso@ucjc.edu 
after the test. Finally, Pruscino et al. ${ }^{(14)}$ investigated the effects of caffeine ingestion $(6 \mathrm{mg} / \mathrm{kg})$ alone or in combination with sodium bicarbonate $(0.3 \mathrm{~g} / \mathrm{kg})$ on a $2 \times 200 \mathrm{~m}$ freestyle time trial. The ingestion of caffeine, alone or combined with sodium bicarbonate, was not effective in reducing the time undertaken to complete the swim test. Therefore, with the available data it is complex to determine whether caffeine or caffeine-containing energy drinks are effective in improving swimming performance.

The aim of this investigation was to determine the effectiveness of a commercially available caffeinated energy drink (3 mg of caffeine/ $\mathrm{kg}$ of body mass) in improving aspects of swimming performance in competitive sprinters. On the basis of previous investigations, we hypothesised that the pre-exercise ingestion of the caffeinated energy drink would reduce the time needed to complete a $50 \mathrm{~m}$ simulated swimming competition.

\section{Methods}

\section{Subjects}

In all, fourteen young male swimmers volunteered to participate in this investigation. They had a mean age of $20 \cdot 2$ (SD $2 \cdot 6$ ) years, height of 183 (SD 7) cm, body mass of 73.9 (SD 8.3) kg and body fat of 8.3 (SD 1.9) \%. We established as inclusion criteria that all the potential participants had obtained the qualifying standards for the 2013 National Spanish Championship in $50 \mathrm{~m}$ swimming competitions, had swimming experience of at least 5 years and had trained at least $6 \mathrm{~d}$ /week during the previous year. For these reasons, we classified this study sample as competitive sprint swimmers. The swimmers were light caffeine consumers (less than one can of soda or energy drink per d), had no previous history of cardiopulmonary diseases and had no musculoskeletal injuries in the previous 3 months. The swimmers were also encouraged to avoid medications or nutritional supplements for the duration of the study. At 1 week before the onset of the study, the participants were fully informed of the experimental standards and the risks and discomforts associated with the research, and gave their informed written consent to participate in the investigation. The study was approved by a Research Ethics Committee in accordance with the latest version of the Declaration of Helsinki.

\section{Experimental design}

A double-blind, placebo-controlled randomised experimental design was used in this study. Each swimmer took part in two experimental trials carried out in the same swimming facility and under the same experimental conditions (ambient temperature $25.3(\mathrm{SD} 1 \cdot 1)^{\circ} \mathrm{C}$; water temperature $27 \cdot 0$ (SD $\left.0 \cdot 1\right)^{\circ} \mathrm{C}$ ). In one trial, swimmers ingested a powdered caffeine-containing energy drink (Fure ${ }^{\circledR}$, ProEnergetics) dissolved in $250 \mathrm{ml}$ of tap water. The amount of energy drink was individually set to provide a dose of $3 \mathrm{mg}$ of caffeine $/ \mathrm{kg}$ of body mass $(3 \mathrm{mg} / \mathrm{kg})$. In another trial, swimmers ingested the same energy drink with no caffeine content (e.g. placebo energy drink; $0 \mathrm{mg} / \mathrm{kg}$ ) but with the same taste. The beverages were ingested $60 \mathrm{~min}$ before the onset of the experimental trials and were provided in opaque plastic bottles to avoid identification. Both experimental beverages also contained taurine $(18.7 \mathrm{mg} / \mathrm{kg})$, sodium bicarbonate $(4.7 \mathrm{mg} / \mathrm{kg})$, L-carnitine $(1.9 \mathrm{mg} / \mathrm{kg})$ and a small amount of carbohydrate $(6.6 \mathrm{mg} / \mathrm{kg}$ in the form of maltodextrin) to sweeten the taste of the drinks. However, the amount of exogenous energy provided in the form of carbohydrate with the beverages was negligible (approximately $8.4 \mathrm{~kJ}$ (2 kcal)). Moreover, these substances were ingested in identical proportions in the two experimental trials. Both experimental trials started at the same time of day (10.00 hours) and were separated by $7 \mathrm{~d}$ to allow for complete recovery and caffeine washout

\section{Experimental protocol}

The swimmers were nude-weighed at $2 \mathrm{~d}$ before the first experimental trial to calculate the amount of energy drink needed to provide an individual a dose of $3 \mathrm{mg} / \mathrm{kg}$ caffeine. In addition, swimmers were encouraged to refrain from all dietary sources of caffeine, alcohol or stimulants for the duration of the study. Before a period of $24 \mathrm{~h}$ of each experimental trial, the swimmers mimicked their habitual routines before competition and thus refrained from strenuous exercise and adopted a precompetition food and fluid regimen. On the day of the experimental trials, the swimmers woke up $4 \mathrm{~h}$ beforehand and had a pre-competition breakfast. Then, they rested until their arrival at the swimming facility. All these standardisations were reported to the technical staff of the team, and food and fluid diaries were obtained and analysed to ensure compliance. In the second experimental trial, all these standardisations were repeated.

At 10.00 hours, the experimental beverages were individually provided in coded bottles. Players ingested the beverage in its entirety and investigators supervised that players drank only from their own labelled bottle. Then, swimmers performed a 45 min standardised warm-up. Just 60 min after the completion of the beverage intake, the swimmers performed two maximal countermovement jumps with 1 min of rest between repetitions. During the jump, participants placed their hands on their waists in order to remove the influence of the arms on jump height. Leg muscle power output during the impulse phase (concentric part of the jump) and jump height were determined using a force platform (Quattro Jump; Kistler). The height of the highest jump was used for statistical analysis.

After $3 \mathrm{~min}$, the swimmers performed a handgrip maximal force production test with both hands (Grip-D). Participants performed two attempts with each hand, with 1 min of recovery between repetitions, and the highest value obtained with each hand was used for analysis. After $5 \mathrm{~min}$ of recovery, the participants performed a simulated $50 \mathrm{~m}$ competition in their habitual swimming style (seven swimmers selected crawl, three selected back stroke, three selected breast stroke and one selected butterfly stroke). The swimmers replicated their pre-competition routines regarding physical and mental preparation. The test was performed in an official $25 \mathrm{~m}$ swimming pool, and swimmers started the test from a homologated platform. The competition was started with an acoustic signal, and swimming time was measured by three experienced trainers using 0.01 
chronometers (Geonaute), as recommended by the International Swimming Federation ${ }^{(15)}$. When two of the three watches recorded the same time and the third disagreed, the two identical times were used as the official test time. When all three watches disagreed, the watch recording the intermediate time was used as the official test time ${ }^{(15)}$. The simulated competition was performed in the central swimming lane, and there were no other swimmers in the pool at the time of the test. Three synchronised video cameras (Sony Handycam HDR-XR200VE; Sony) were placed at the start, at $12.5 \mathrm{~m}$ from the start line and at the end of the pool to analyse stroke frequency and stroke length during the whole test.

After 10 min of recovery, the swimmers performed a $45 \mathrm{~s}$ maximal swimming test in a swim ergometer (Vasa Ergometer; Vasa). For this test, participants lay prone on a bench and replicated the upper-body movements during breast stroke. The resistance during the simulated swimming was provided by an airflow resistance system connected with metal wires to paddles, which were adjusted to the swimmers hands. For this test, the swimmers started in a stationary position and swam as fast as possible against the resistance produced in the paddles. The power produced during each stroke, the stroke rate and the heart rate (Polar) were instantaneously recorded and analysed afterwards. Maximal and mean values during this test were used for analysis. After $1 \mathrm{~min}$ of the end of the ergometer test, a capillary blood sample was obtained from a fingertip to assess blood lactate concentration (Lactate Pro; Arkray Global Business).

Then, the swimmers were required to fill out a questionnaire about their sensations of power, endurance and perceived exertion during the swimming-specific tests. This questionnaire included a one- to ten-point scale to assess each item, and participants were previously informed that one point meant a minimal amount of that item and ten points meant a maximal amount of the item. This questionnaire has been used previously to assess subjective feelings of power, endurance and fatigue in other sport situations ${ }^{(9)}$. In addition, participants were provided with a survey to be filled out the following morning about sleep quality, nervousness, gastrointestinal problems and other discomforts. This survey included six items on a yes/no scale, and it has been used previously to assess the side effects derived from energy drink ingestion ${ }^{(11)}$. This survey also included specific questions to evaluate the success of the participant blinding procedure.

\section{Statistical analysis}

Normality was tested for each variable with the Shapiro-Wilk test. All the variables included in this investigation presented a normal distribution $(P>0.05)$, and parametric statistics were used to determine the effectiveness of the caffeinated energy drink to increase swimmers' performance. Differences between the caffeinated $v$. placebo energy drink in the variables obtained once in each experimental trial (e.g. $50 \mathrm{~m}$ competition time, subjective feelings, blood lactate concentration and so on) were determined using paired $t$ tests. Differences between the caffeinated $v$. placebo energy drink in the variables obtained twice or more in each experimental trial (power production and heart rate during the swim ergometer test and so on) were determined by two-way ANOVA (beverage $\times$ time) with repeated measures. After a significant $F$ test (Geisser-Greenhouse correction for the assumption of sphericity), differences between means were identified using Tukey's HSD post hoc. Differences on side effects after beverage intake were analysed using the McNemar test. The data were analysed with the statistical package SPSS version 20.0 (SPSS Inc.). The results are presented as mean values and standard deviations for fourteen sprint swimmers. The significance level was set at $P<0.05$.

\section{Results}

In comparison with the placebo energy drink, the pre-exercise ingestion of the caffeinated energy drink increased the jump height during a countermovement jump by 3.0 (SD $7 \cdot 0$ ) \% $(P<0 \cdot 05$, Table 1$)$, although the increase in leg muscle power output during the jump obtained with the caffeinated energy drink did not reach statistical significance. The caffeinated energy drink increased handgrip force in the right hand by 3.5 (sD 4.9$) \%(P<0.05$; Table 1$)$, although the change in the left hand was not statistically significant. During the $50 \mathrm{~m}$ simulated competition, swimmers reduced their time by 0.9 (SD 1.5 ) \% when they ingested the caffeinated energy drink in comparison with the placebo energy drink $(P<0.05$; Table 1$)$. However, there were no differences in stroke frequency (1.30 (SD 0.43) $v$. 1.29 (sD 0.47) strokes/s; $P=0.92$ ) or stroke length (1.58 (sD 0.55) $v .1 .59(\mathrm{sD} 0.61) \mathrm{m} /$ stroke; $P=0.62)$ between experimental trials. Peak power during the $45 \mathrm{~s}$ swim ergometer test was $11 \cdot 2$

Table 1. Physical performance in swimming-specific testing with the ingestion of a caffeinated energy drink ( $3 \mathrm{mg}$ of caffeine/kg of body weight) or the same drink without caffeine (placebo drink)

(Mean values and standard deviations; fourteen competitive sprint swimmers)

\begin{tabular}{|c|c|c|c|c|c|c|c|}
\hline \multirow[b]{2}{*}{ Variables } & \multicolumn{2}{|c|}{ Placebo drink } & \multicolumn{2}{|c|}{ Energy drink } & \multicolumn{2}{|c|}{$\Delta(\%)$} & \multirow[b]{2}{*}{$P$} \\
\hline & Mean & SD & Mean & SD & Mean & SD & \\
\hline Jump height $(\mathrm{cm})$ & 49.4 & $5 \cdot 3$ & 50.9 & $5 \cdot 2$ & $3 \cdot 0$ & $7 \cdot 0$ & 0.04 \\
\hline Leg power output (W/kg) & 27.9 & 3.4 & $28 \cdot 3$ & $3 \cdot 3$ & 1.1 & $6 \cdot 0$ & 0.36 \\
\hline Right handgrip force $(\mathrm{N})$ & 481 & 49 & 498 & 43 & 3.5 & 4.9 & 0.01 \\
\hline Left handgrip force $(\mathrm{N})$ & 467 & 55 & 475 & 66 & 1.8 & 3.7 & 0.34 \\
\hline 50 m simulated swimming competition (s) & 27.77 & 3.43 & 27.45 & 3.21 & -0.9 & 1.5 & 0.01 \\
\hline Peak power swim ergometer (W) & 273 & 55 & 303 & 49 & $11 \cdot 2$ & 5.4 & 0.04 \\
\hline Mean power swim ergometer (W) & 201 & 35 & 210 & 21 & 4.9 & $7 \cdot 6$ & 0.08 \\
\hline Peak heart rate swim ergometer (beats/min) & 166 & 1 & 169 & 1 & $2 \cdot 6$ & 6.9 & 0.28 \\
\hline Blood lactate concentration (mм) & 11.00 & 1.97 & 11.67 & $20 \cdot 60$ & $6 \cdot 0$ & 3.2 & 0.04 \\
\hline
\end{tabular}


(SD 5.4) \% higher with the caffeinated energy drink than with the placebo energy drink $(P<0.05)$, and there was a tendency towards increased mean power during this test $(P=0.08$; Table 1). Fig. 1 depicts averaged swimming power at 5-s intervals during the ergometer test. Although swimming power with the caffeinated energy drink was greater than with the placebo drink during the entire test, the differences were only significant at the end of the test $(P<0 \cdot 05$; Table 1$)$. Blood lactate concentration at the end of the swim ergometer test was $6 \cdot 0$ (sD $3.2) \%(P<0.05)$ higher with the caffeinated energy drink than with the placebo drink, but there were no differences in the peak heart rate obtained during the test.

Fig. 2 depicts the individual changes produced by the ingestion of the caffeinated energy drink (when compared with the values obtained with the placebo drink) in all the performance tests carried out in this investigation. Positive values indicate that performance was superior with the caffeinated energy drink $v$. the placebo drink. Although most participants presented a positive response to the ingestion of the caffeinated energy drink in one or several tests, participant 6 did not benefit from caffeine ingestion while participants 7 and 12 presented a clear ergolytic effect in most tests.

The subjective feelings of muscle power during the swimming-specific tests were increased after the ingestion of the caffeinated energy drink in comparison with the placebo $(P<0.05$; Table 2$)$. However, self-reported endurance fitness and fatigue after the testing were similar with the two experimental beverages. Furthermore, the prevalence of side effects in the hours following the ingestion of the experimental beverages was similar with the caffeinated and placebo energy drinks (Table 2). Only $36 \%$ (five out of fourteen) of participants correctly guessed the order of the trials, indicating successful blinding of the participants to the interventions.

\section{Discussion}

The aim of this investigation was to determine the efficacy of a caffeinated energy drink (in an amount equivalent to providing $3 \mathrm{mg} / \mathrm{kg}$ of caffeine) to improve aspects of sprint swimming performance. For this purpose, fourteen competitive swimmers performed several swimming-specific tests and a $50 \mathrm{~m}$ simulated competition after the ingestion of the caffeinated energy drink or the same drink without caffeine. The pre-exercise ingestion of the caffeinated energy drink increased jump height during a countermovement jump, improved handgrip force in the right hand and augmented peak power during a $45 \mathrm{~s}$ swim ergometer test (Table 1). In addition, the caffeinated drink reduced the time needed to complete the $50 \mathrm{~m}$ simulated swimming competition (Table 1). Although these ergogenic effects were accompanied by increased self-perception of muscle power, the caffeinated energy drink did not increase the prevalence of side effects typically found after the ingestion of caffeine-containing products (Table 2). All this information suggests that caffeinated energy drinks are a potential ergogenic aid to increase aspects of physical performance in competitive sprint swimmers.

Despite the abundant amount of scientific information about the ergogenicity of caffeine or caffeine-containing products for

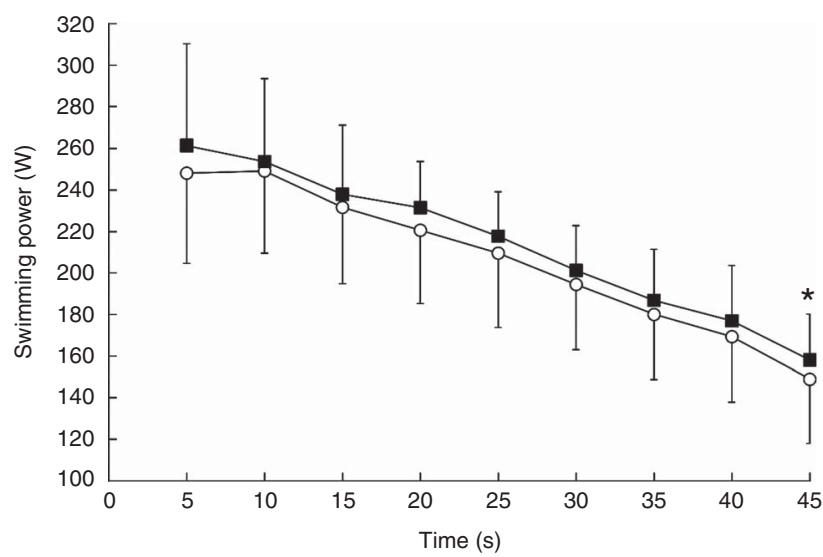

Fig. 1. Swimming power production during a $45 \mathrm{~s}$ test on a swim ergometer with the ingestion of a caffeinated energy drink ( $3 \mathrm{mg}$ of caffeine $/ \mathrm{kg}$ of body weight) or the same drink without caffeine (placebo drink). Data are means for fourteen sprint swimmers, with standard deviations represented by vertical bars. *Different from the placebo drink $(P<0.05)$. $-\bigcirc-$ Placebo drink; - energy drink.

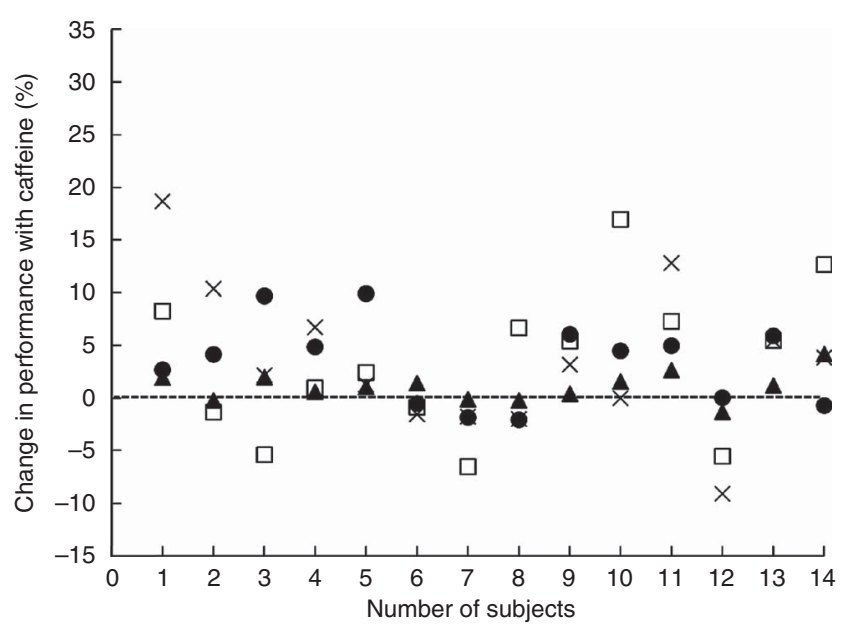

Fig. 2. Changes in swimming-specific performance with the ingestion of a caffeinated energy drink ( $3 \mathrm{mg}$ of caffeine/ $\mathrm{kg}$ of body weight) $v$. the same drink without caffeine (placebo drink). Each data point represents the individual change in performance with the caffeinated energy drink with respect to the placebo drink on handgrip force (an averaged change of the two hands), jump height, $50 \mathrm{~m}$ swimming time and swimming power. $\mathrm{C}$, Handgrip force; $\square$, jump height; $\Lambda, 50 \mathrm{~m}$ performance; $X$, swimming power.

physical performance in endurance disciplines ${ }^{(16)}$ and team sports $^{(17)}$, only a few investigations have been geared to determining the ergogenicity of caffeine for swimming performance ${ }^{(12-14)}$. Moreover, the results of these latter investigations are inconclusive to determine the ergogenic effects of caffeine on swimming performance because of differences in the swimming test used $(2 \times 100 \mathrm{~m}$ with $20 \mathrm{~min}$ of passive recovery v. $1500 \mathrm{~m}$ time trials), the dosage of caffeine administered (an absolute $250 \mathrm{mg}$ dose $v$. individualised $6 \mathrm{mg} / \mathrm{kg}$ doses) and the combination with other substances. The present investigation offers new information on this topic because the results indicate that the ingestion of $3 \mathrm{mg} / \mathrm{kg}$ caffeine in the form of an energy drink improved physical performance in several physical tests and reduced the time needed to complete a $50 \mathrm{~m}$ swimming 
Table 2. Rates of perceived fitness during the swimming-specific testing and prevalence of side effects in the following hours after the ingestion of a caffeinated energy drink ( $3 \mathrm{mg}$ of caffeine $/ \mathrm{kg}$ of body weight) or the same drink without caffeine (placebo drink) (Mean values and standard deviations; fourteen competitive sprint swimmers)

\begin{tabular}{|c|c|c|c|c|c|}
\hline \multirow[b]{2}{*}{ Items } & \multicolumn{2}{|c|}{$\begin{array}{l}\text { Placebo } \\
\text { drink }\end{array}$} & \multicolumn{2}{|c|}{$\begin{array}{l}\text { Energy } \\
\text { drink }\end{array}$} & \multirow[b]{2}{*}{$P$} \\
\hline & Mean & $S D$ & Mean & SD & \\
\hline Power & 6 & 1 & 7 & 1 & 0.02 \\
\hline Endurance & 6 & 2 & 6 & 2 & 0.50 \\
\hline Exertion & 6 & 2 & 7 & 1 & 0.60 \\
\hline Headache (\%) & 0 & & 7 & & 0.31 \\
\hline Abdominal/gut discomfort (\%) & 7 & & 7 & & 1.00 \\
\hline Muscle soreness (\%) & 36 & & 36 & & 1.00 \\
\hline Increased vigour/activeness (\%) & 0 & & 0 & & 1.00 \\
\hline Tachycardia and heart palpitations (\%) & 0 & & 0 & & 1.00 \\
\hline Insomnia (\%) & 7 & & 7 & & 1.00 \\
\hline Increased anxiety (\%) & 7 & & 0 & & 0.31 \\
\hline
\end{tabular}

competition. In fact, the results of the jump test suggest that caffeine might confer some advantage during the start of the competition (Table 1), whereas the results of the swim ergometer test indicate that the application of effective power during swimming was improved with the caffeinated energy drink (Fig. 1). Previous and current outcomes suggest the ergogenicity of caffeine for swimming, at least in short-duration and high-intensity trials.

Apart from the objective data obtained during the physical testing, the sprint swimmers also reported increased selfperceived muscle power after the ingestion of the caffeinated energy drink (Table 2), although the feelings of endurance and fatigue were similar between beverages. The improved selfperception of physical fitness after the ingestion of caffeinated energy drinks has been previously reported in basketball ${ }^{(6)}$, soccer $^{(5)}$, volleyball $^{(7)}$ and rugby players ${ }^{(9)}$. Accompanying the increased feelings of fitness, most investigations have also found that athletes report increased activeness and nervousness and a higher prevalence of insomnia during the hours following the ingestion of caffeinated drinks ${ }^{(5,7,9)}$, as previously reported with other caffeine-containing beverages ${ }^{(18)}$.

Although caffeine was removed from the World Antidoping Agency (WADA) list of doping substances in $2004^{(3)}$, national and international antidoping authorities are still concerned about the use and misuse of this substance in sports. Caffeine has been periodically included in the WADA Monitoring Program $^{(4)}$ to assess the use of this substance during sport competitions and to determine the adequacy of its future re-inclusion in the doping list based on the prevalence of side effects derived from the use of caffeinated products. Previous investigations have determined that the ingestion of the caffeinated energy drinks (with $3 \mathrm{mg} / \mathrm{kg}$ of caffeine) increased the prevalence of side effects such as insomnia ${ }^{(5,7,19)}$, vigour and activeness $^{(6,11)}$. However, in the present investigation with sprint swimmers, activeness and insomnia were rated similarly after the ingestion of the caffeinated energy drink and the placebo drink. The absence of these side effects after the ingestion of the caffeinated beverage was probably because all the experimental trials were performed in the morning, whereas previous investigations were carried out with an administration of caffeinated beverages in the afternoon ${ }^{(5-7,11,19)}$. In any case, the low prevalence of side effects derived from the ingestion of $3 \mathrm{mg} / \mathrm{kg}$ caffeine in the form of an energy drink does not preclude the recommendation of these beverages to improve swimming performance in sprint swimmers. Although it was not included in this investigation, it is necessary to investigate the impact of the chronic use of caffeinated energy drinks on both performance and side effects, especially for the vulnerable populations such as children ${ }^{(20)}$.

Previous investigations have suggested a number of explanations for the ergogenicity derived from caffeine ingestion. Initially, it was believed that caffeine enhanced free fatty acid oxidation and consequently helped to spare muscle and liver glycogen $^{(21)}$. However, it seems unlikely that this theory explains the ergogenicity found in the present investigation because all tests lasted $45 \mathrm{~s}$ or less and muscle and liver glycogen were not depleted. Other suggested mechanisms for caffeine ergogenicity are the enhanced calcium mobilisation in the sarcoplasmic reticulum, the inhibition of phosphodiesterase or the enhanced $\mathrm{Na}^{+} / \mathrm{K}^{+}$pump activity, although all these peripheral mechanism are rarely found in vivo and with nor$\mathrm{mal} /$ physiological plasma caffeine concentrations ${ }^{(22)}$. The most recent investigations indicate that caffeine might improve sports performance via enhanced central drive rather than peripheral changes ${ }^{(21)}$. On the basis of this hypothesis, caffeine acts antagonistically on adenosine receptors and inhibits the negative effects of adenosine on neural drive ${ }^{(23)}$. Although this hypothesis has been mainly tested in animals, investigations with humans have determined an improved voluntary contraction $^{(24)}$ and better intra- and inter-muscular coordination during muscle contractions ${ }^{(11)}$ after the ingestion of $3-6 \mathrm{mg} / \mathrm{kg}$ caffeine.

In the present investigation, we did not assess the origin of the ergogenicity obtained with the ingestion of the caffeinated energy drink. However, we found that sprint swimmers presented higher blood lactate concentrations after the swim ergometer test with the caffeinated energy drink, as compared with the placebo drink (Table 1). This same effect on blood lactate concentration has been found after a $2 \times 100 \mathrm{~m}$ swimming time trial with $250 \mathrm{mg}$ of caffeine ${ }^{(12)}$, whereas an increased blood glucose concentration has also been reported in swimmers after the ingestion of $6 \mathrm{mg} / \mathrm{kg}$ caffeine ${ }^{(13)}$. All these data suggest that the enhanced aspects of performance obtained with caffeinated products in sprint swimmers was related to a higher utilisation of the anaerobic (e.g. glycolysis) pathways.

The effect of caffeine on exercise performance has been typically presented as a group mean. However, a few studies have reported caffeine effects individually during short-term activities ${ }^{(25-29)}$. In these studies, most but not all individuals presented better performances after the ingestion of $2-6 \mathrm{mg} / \mathrm{kg}$ caffeine, but there were individuals with minimal ergogenic effects or even slight ergolytic effects (e.g. 'non-caffeine responders'). Similarly, our investigation presented positive performance effects on most but not all sprint swimmers (eleven out of fourteen swimmers), whereas three swimmers did not increase performance in any of the test with the caffeinated energy drink ingestion (Fig. 2). The physiological 
causes for a lack of ergogenic response to caffeine ingestion in some individuals have not been completely identified, but they might be related to the cytochrome $\mathrm{P} 450$ gene (CYP1A2). Briefly, the effects of caffeine in several physiological responses are different in individuals with the $\mathrm{C}$ variant $v$. the A variant of the CYP1A2 polymorphism ${ }^{(30)}$. Individuals who are AA homozygous for the CYP1A2 allele are considered 'rapid' caffeine metabolisers, whereas carriers of the $\mathrm{C}$ variant are 'slow' caffeine metabolisers ${ }^{(31)}$. In fact, individuals homozygous for the A allele of this polymorphism presented higher ergogenic effects with the ingestion of $6 \mathrm{mg} / \mathrm{kg}$ caffeine before a simulated $40 \mathrm{~km}$ time trial, as compared with $\mathrm{C}$ allele carriers ${ }^{(29)}$. Future studies are necessary to establish the impact of the CYP1A2 polymorphism on caffeine metabolism and ergogenicity during exercise. However, our results suggest the existence of innate differences in the performance response to caffeine ingestion.

A limitation of the present investigation is the use of manual timing to assess swimming times during the simulated $50 \mathrm{~m}$ competition instead of using automatic officiating equipment. Although swimming times were measured by three experienced trainers using $0.01 \mathrm{~s}$ chronometers, as recommended by the International Swimming Federation ${ }^{(15)}$, the use of manual timing implies a loss of sensitivity and reliability for the assessment of swimming performance. In addition, some important information of interest (e.g. reaction time off the blocks) has not been analysed in this investigation. A second limitation of the investigation is the co-ingredients included in the experimental drinks (both the caffeinated one and the placebo). The experimental drinks contained not only caffeine but also slight amounts of carbohydrate, taurine, sodium bicarbonate and L-carnitine. Because these components were included in the same proportions in both experimental drinks, we suggest that caffeine is the only substance responsible for the effects obtained with the caffeinated energy drink. However, more information is necessary to elucidate whether the co-ingestion of these substances is necessary to obtain the purported benefits of caffeine.

\section{Conclusions}

In summary, the ingestion of a commercially available energy drink, with $3 \mathrm{mg}$ of caffeine $/ \mathrm{kg}$ of body mass, was effective to increase swimming peak power during a $45 \mathrm{~s}$ swim ergometer test and to reduce the time needed to complete a $50 \mathrm{~m}$ simulated competition. These outcomes suggest that swimming performance can be increased by using moderate doses of caffeine rather the larger doses $(>6 \mathrm{mg} / \mathrm{kg})$ often used by swimmers and other athletes ${ }^{(3)}$. Moreover, the ingestion of the caffeinated energy drink did not increase the rate of typical side effects found after a swimming competition. The low prevalence of side effects is particularly important in a sport like swimming in which major competitions require multiple performances over several days. Although the ergogenicity of the caffeinated energy drink was present in most participants in this investigation, some of them (three out of fourteen) did not benefit from the energy drink intake. Thus, caffeinated energy drinks are a potential ergogenic aid to increase aspects of physical performance for most competitive sprint swimmers.

\section{Acknowledgements}

The authors thank the participants for their invaluable contribution to the study. The authors also want to acknowledge the work of Juan Camus in recruiting the participants.

Contributions of authors were as follows: B. L. and J. D. C. formulated the research question; B. L., D. R.-V., F. A., J. A.-V., J. J. S., C. G.-M., C. G.-S. and J. D. C. designed the study; B. L., D. R.-V., F. A., J. A.-V., J. J. S., C. G.-M., C. G.-S. and J. D. C. carried out the study; B. L., J. J. S. and J. D. C. analysed the data; B. L., J. J. S. and J. D. C. wrote the article; D. R.-V., F. A., C. G.-M. and C. G.-S. revised the article.

This study did not receive any funding. The energy drinks were provided by Fure (ProEnergetics, Spain).

The authors declare that they have no conflict of interest derived from the outcomes of this study.

\section{References}

1. Del Coso J, Munoz-Fernandez VE, Munoz G, et al. (2012) Effects of a caffeine-containing energy drink on simulated soccer performance. PLOS ONE 7, e31380.

2. Hoyte CO, Albert D \& Heard KJ (2013) The use of energy drinks, dietary supplements, and prescription medications by United States college students to enhance athletic performance. J Community Health 38, 575-580.

3. Del Coso J, Munoz G \& Munoz-Guerra J (2011) Prevalence of caffeine use in elite athletes following its removal from the World Anti-Doping Agency list of banned substances. Appl Physiol Nutr Metab 36, 555-561.

4. World Antidoping Agency (2015) Monitoring program. https://wada-main-prod.s3.amazonaws.com/resources/files/ wada-2015-monitoring-program-en.pdf (accessed 1 January 2015).

5. Lara B, Gonzalez-Millan C, Salinero JJ, et al. (2014) Caffeinecontaining energy drink improves physical performance in female soccer players. Amino Acids 46, 1385-1392.

6. Abian-Vicen J, Puente C, Salinero JJ, et al. (2014) A caffeinated energy drink improves jump performance in adolescent basketball players. Amino Acids 46, 1333-1341.

7. Del Coso J, Perez-Lopez A, Abian-Vicen J, et al. (2014) Enhancing physical performance in male volleyball players with a caffeine-containing energy drink. Int J Sports Physiol Perform 9, 1013-1018.

8. Del Coso J, Ramirez JA, Munoz G, et al. (2013) Caffeinecontaining energy drink improves physical performance of elite rugby players during a simulated match. Appl Physiol Nutr Metab 38, 368-374.

9. Del Coso J, Portillo J, Munoz G, et al. (2013) Caffeinecontaining energy drink improves sprint performance during an international rugby sevens competition. Amino Acids $\mathbf{4 4}$, 1511-1519.

10. Astorino TA, Matera AJ, Basinger J, et al. (2012) Effects of red bull energy drink on repeated sprint performance in women athletes. Amino Acids 42, 1803-1808.

11. Del Coso J, Salinero JJ, Gonzalez-Millan C, et al. (2012) Dose response effects of a caffeine-containing energy drink on muscle performance: a repeated measures design. J Int Soc Sports Nutr 9, 21.

12. Collomp K, Ahmaidi S, Chatard JC, et al. (1992) Benefits of caffeine ingestion on sprint performance in trained and untrained swimmers. Eur J Appl Physiol Occup Physiol 64, 377-380. 
13. MacIntosh BR \& Wright BM (1995) Caffeine ingestion and performance of a 1,500-metre swim. Can J Appl Physiol 20, 168-177.

14. Pruscino CL, Ross ML, Gregory JR, et al. (2008) Effects of sodium bicarbonate, caffeine, and their combination on repeated 200-m freestyle performance. Int J Sport Nutr Exerc Metab 18, 116-130.

15. International Swimming Federation (2014) FINA swimming rules. http://www.fina.org/H2O/docs/rules/FINAswrules_20132017. pdf (accessed 1 April 2014).

16. Burke LM (2008) Caffeine and sports performance. Appl Physiol Nutr Metab 33, 1319-1334.

17. Holway FE \& Spriet LL (2011) Sport-specific nutrition: practical strategies for team sports. J Sports Sci 29, Suppl. 1, S115-S125.

18. Dorea JG \& da Costa TH (2005) Is coffee a functional food? Br J Nutr 93, 773-782.

19. Abian P, Del Coso J, Salinero JJ, et al. (2014) The ingestion of a caffeinated energy drink improves jump performance and activity patterns in elite badminton players. J Sports Sci 33, 1042-1050.

20. Ruxton CH (2014) The suitability of caffeinated drinks for children: a systematic review of randomised controlled trials, observational studies and expert panel guidelines. J Hum Nutr Diet 27, 342-357.

21. Davis JK \& Green JM (2009) Caffeine and anaerobic performance: ergogenic value and mechanisms of action. Sports Med 39, 813-832.

22. Magkos F \& Kavouras SA (2005) Caffeine use in sports, pharmacokinetics in man, and cellular mechanisms of action. Crit Rev Food Sci Nutr 45, 535-562.
23. Davis JM, Zhao Z, Stock HS, et al. (2003) Central nervous system effects of caffeine and adenosine on fatigue. $A m J$ Physiol Regul Integr Comp Physiol 284, R399-R404.

24. Del Coso J, Estevez E \& Mora-Rodriguez R (2008) Caffeine effects on short-term performance during prolonged exercise in the heat. Med Sci Sports Exerc 40, 744-751.

25. Doherty M (1998) The effects of caffeine on the maximal accumulated oxygen deficit and short-term running performance. Int J Sport Nutr 8, 95-104.

26. Doherty M, Smith P, Hughes M, et al. (2004) Caffeine lowers perceptual response and increases power output during highintensity cycling. J Sports Sci 22, 637-643.

27. Skinner TL, Jenkins DG, Coombes J, et al. (2010) Dose response of caffeine on 2000-m rowing performance. Med Sci Sports Exerc 42, 571-576.

28. Wiles JD, Coleman D, Tegerdine M, et al. (2006) The effects of caffeine ingestion on performance time, speed and power during a laboratory-based $1 \mathrm{~km}$ cycling time-trial. J Sports Sci 24, 1165-1171.

29. Womack CJ, Saunders MJ, Bechtel MK, et al. (2012) The influence of a CYP1A2 polymorphism on the ergogenic effects of caffeine. J Int Soc Sports Nutr 9, 7.

30. Hallstrom H, Melhus H, Glynn A, et al. (2010) Coffee consumption and CYP1A2 genotype in relation to bone mineral density of the proximal femur in elderly men and women: a cohort study. Nutr Metab (Lond) 7, 12.

31. Cornelis MC, El-Sohemy A, Kabagambe EK, et al. (2006) CYP1A2 genotype, and risk of myocardial infarction. JAMA 295, 1135-1141. 